\title{
Supersymmetry theory of microphase separation in homopolymer-oligomer mixtures
}

\author{
Alexander Olemskoi, ${ }^{*}$ Ivan Krakovsky, ${ }^{\dagger}$ and Alexey Savelyev ${ }^{\ddagger}$ \\ Physical and Technical Department, Sumy State University, Rimskii-Korsakov Street 2, 40007 Sumy, Ukraine \\ and Department of Macromolecular Physics, Charles University V Holešovičkách 2, 18000 Prague 8, Czech Republic
}

(Received 4 August 2003; published 17 February 2004)

\begin{abstract}
The mesoscopic structure of periodically alternating layers of stretched homopolymer chains surrounded by perpendicularly oriented oligomeric tails is studied for systems with both strong (ionic) and weak (hydrogen) interactions. We focus on the consideration of the distribution of oligomers along the homopolymer chains that is described by the effective equation of motion with the segment number playing the role of imaginary time. The supersymmetry technique is developed to consider associative hydrogen bonding, self-action effects, inhomogeneity, and temperature fluctuations in the oligomer distribution. Making use of the self-consistent approach allows one to explain experimentally observed temperature dependence of the structure period and the order-disorder transition temperature and period as functions of the oligomeric fraction for systems with different bonding strengths. A whole set of parameters of the model used is found for strong, intermediate, and weak coupled systems being Poly (4-vinyl pyridine)-dodecyl benzene sulfonic acid [P4VP-(DBSA) $)_{x}$, P4VP$\left[\mathrm{Zn}(\mathrm{DBS})_{2}\right]_{x}$, and P4VP- 3-pentadecyl Phenol $x$, respectively. A passage from the former two to the latter is shown to cause a crucial decrease in the magnitude of both parameters of hydrogen bonding and self-action, as well as the order-disorder transition temperature.
\end{abstract}

DOI: $10.1103 /$ PhysRevE.69.021803

PACS number(s): 36.20.-r, 64.60.Cn, 11.30.Pb

\section{INTRODUCTION}

Surfactant-induced mesomorphic structures based on the association between flexible homopolymers and headfunctionalized oligomers represent a new class of supramolecular materials. They exhibit a rich phase behavior due to which they have attracted, during the past decade, considerable attention of both experimentalists [1-5] and theoreticians $[6,7]$. Microphase separation is the principal property of such systems which results in the formation of ordered mesoscopic structures due to the association between the head group of the oligomer and the corresponding groups of the homopolymer, on the one hand, and unfavorable polarnonpolar interactions between the nonpolar tail of the surfactant molecules and the rest of the system, on the other hand.

The homopolymer-oligomer systems involve two main classes that are relevant to strong ionic bonds and weak hydrogen ones. Unlike conventional copolymers where repulsive blocks are bonded together by covalent bonds, there are various temporary physical interactions which play a crucial role in the formation of ordered mesophases in such systems. In the ionic bonding systems the degree of association is relatively high, so that the polymer chain resembles a comb copolymer with regularly alternating oligomer side chains. At the same time, for the systems with temperaturedependent hydrogen bonds the incompatibility must not be so strong to induce separation on a macroscopic level. Here, the microphase separation results in the periodic alternation of the layers of stretched homopolymer chains surrounded by perpendicularly oriented oligomer tails (see Fig. 1). Similarly to the conventional copolymer systems, a rich variety of

\footnotetext{
*Electronic address: olemskoi@ssu.sumy.ua

${ }^{\dagger}$ Electronic address: ivank@kmf.troja.mff.cuni.cz

†Electronic address: alexsav@kmf.troja.mff.cuni.cz
}

morphologies (lamellar, cylindrical, spherical, etc.) is shown to be possible [1]. However, for the sake of simplicity we will restrict ourselves to lamellar morphology.

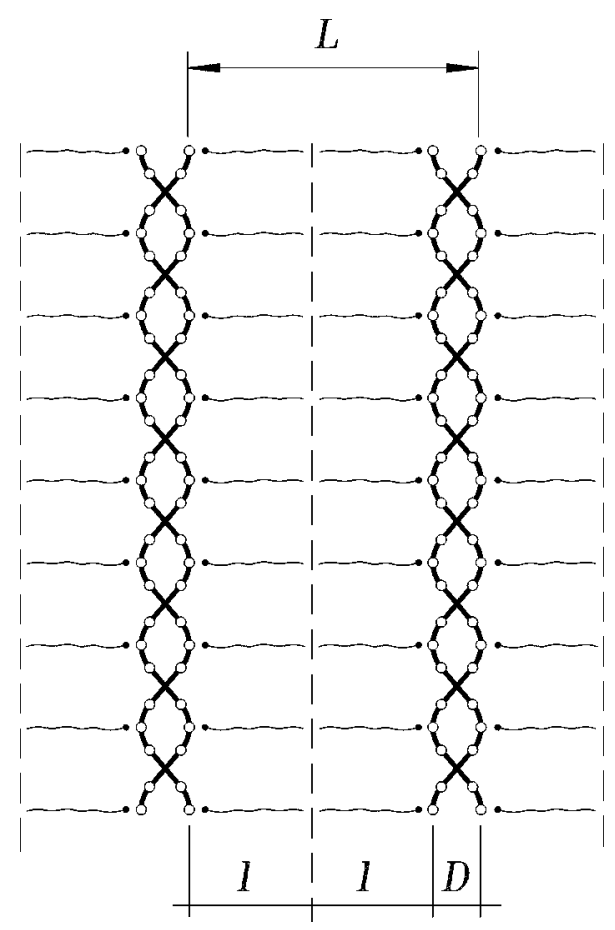

FIG. 1. Schematic picture of the homopolymer-oligomer microphase separated structure (fluctuations of periodicity are ignored). The flexible homopolymer chains are pictured as thick curves with functionalized groups (whole circles) where the head groups of the oligomers (thin tails with heads) are attached. The structure is evolved along the vertical axis with period $L=2 l+D$ determined by the oligomer length $l$ and the thickness $D$ of the homopolymer layer. The number of oligomer groups per homopolymer ring is put to be $x=1 / 3$. 
An example of the ionic bonding systems is the mixture P4VP-(DBSA $)_{x}$ of the homopolymer atactic poly(4-vinyl pyridine) (P4VP) and the surfactant dodecyl benzene sulfonic acid (DBSA). Here, owing to the very strong interaction, the temperature domain of microphase separation is not bounded from above by association effects $[1,2]$. The peculiarity of the systems of this type, being polyelectrolytesurfactant complexes, is that the long space structure period is an increasing function of the number oligomer/monomer ratio $x$ (the number of DBSA groups per pyridine ring). A more complicated behavior is inherent in the hydrogen bonded systems which were considered to study the opposite weak-bonding limit [3-5]. Here, the weak interaction causes an order-disorder transition to a homogeneous hightemperature state. An example of these systems is given by the mixture P4VP-(PDP) $)_{x}$ of the same homopolymer P4VP with 3-pentadecyl phenol (PDP) being the oligomer. In this case, unlike in the ionic bonding systems, the long space period decreases while $x$ increases. Intermediate behavior is exhibited by the system P4VP-[Zn(DBS $\left.)_{2}\right]_{x}$ with the oligomer being zinc dodecyl benzene sulfonate $\mathrm{Zn}(\mathrm{DBS})_{2}$, which forms transition metal coordination complexes with the monomers of P4VP [2]. Ionic bond weakening due to the absence of covalently bound charges along the homopolymer chain leads here to a nonmonotonic form of the $x$ dependence of the long space period.

Principally important for our consideration is the decreasing form of the temperature dependence of the long space period for all the above systems [2-5]. However, such character of the dependence appears in hydrogen bonded systems only within a finite temperature interval bounded by the glass transition temperature $T_{g}$ from below and order-disorder transition temperature $T_{c}$ from above [3,4]. Here, an increase of the oligomer/monomer ratio $x$ leads to a nonmonotonic behavior of the temperature $T_{c}$ with a maximum near the point $x=0.85$, deviation from which narrows the temperature domain $T_{g}-T_{c}$. This domain is the region of our interest where a purely microphase separated structure is possible. Below the glass transition temperature $T_{g}$ the crystallization of the oligomer chains occurs that causes a reduction of the overall volume of the system and a sudden decrease of the long space period [4].

Microphase separation phenomenon had been extensively studied in the past two decades for a variety of polymer systems including random heteropolymers [8-10]. Theoretical studies of the homopolymer-oligomer mixtures, being the systems of associating polymers, were proposed by Tanaka et al. [6] and Dormidontova et al. [7] within the random phase approximation introduced by Leibler [8]. Here, the total free energy

$$
F_{t o t}=F_{h o}+F_{h b}
$$

is written as a sum of two terms, $F_{h o}$ related to the nonassociated homopolymer-oligomer mixture and $F_{h b}$ attributed to the hydrogen bonding. Then, making use of minimization principle with respect to the dependence of the free energy $F_{t o t}$ on the average fraction of hydrogen bonds $X$ present in the system, permits to find the temperature dependence $X(T)$ and to study possible forms of phase diagrams for both macrophase and microphase separations. It turned out that this approach gives the real dependence of the long space period $L$ of the ordered structure on the oligomer/monomer ratio in the system, however, as the fraction of hydrogen bonds monotonically decreases with increase in temperature, the increasing temperature dependence of $L(T)$ obtained is in contradiction to the experimental data [4]. This inconsistency is caused obviously by the roughness of the random phase approximation used for description of the hydrogen bonding.

To avoid this limitation, our approach is based on the above mentioned analogy between associating homopolymer-oligomer mixtures and random comb copolymers taking into account the varying number of oligomers attached to the main chain stochastically. Such a system can be analyzed in terms of the random walk statistics to apply the field theoretical scheme [11] for the development of the microscopic theory. The cornerstone of our approach lies in the assumption that the alternation of the homopolymer associative groups with and without oligomers attached is like the alternation of the segments of different types along the chains of a random heteropolymer to be represented as a stochastic variation of the Ising spin, for which the role of imaginary time is played by the number of chain segments $n$ [12-14].

Along this line, the problem under consideration is divided into two parts, the first of which is reduced to the determination of the relation between the long space period $L$ and the average fraction of hydrogen bonds $X$, whereas the second one is focused on the determination of the frequency $\omega=2 \pi X$ in the distribution of the oligomer heads along the homopolymer chain. The first part of the problem was studied on the basis of the simplest model [5] that is reduced to the treatment of the dependence $F_{h o}(L)$ given by the first term of the free energy (1). Corresponding consideration developed within the framework of the strong segregation limit derives to generic relation (A8) for the dependence $L(\omega)$ (see Appendix A). In this paper, we focus on the second problem to be related to the definition of an optimal frequency $\omega$ that minimizes the second term of the free energy (1) within the framework of the weak segregation limit.

The formal basis of our treatment is the field theoretical scheme of stochastic systems, making use of the supersymmetry field [11]. Conformably to the polymers, this theory was proposed in Ref. [15] and developed for the random copolymers in Refs. [12-14]. Our approach is based on the Martin-Siggia-Rose method of the generating functional [16]. Power and generality of the supersymmetry field scheme were demonstrated for the Sherrington-Kirkpatrick model for which they are identical to the replica approach [17]. The formal basis of the supersymmetry is a nilpotent quantity which represents a square root of 0 . In this sense, the superfield is similar to the complex field, in which the imaginary unit, being square root of -1 , is used instead of the anticommuting nilpotent quantity being the Grassmann variable. By definition, the supersymmetry field combines the commuting boson and anticommuting fermion components into the unified mathematical construction representing a vector in the supersymmetry space. Choice of the optimal 
basis of the supersymmetry correlator yields in optimal way the advanced/retarded Green functions and the structure factor to obtain microscopic expression for the frequency $\omega$.

The paper is organized in the following manner. Section II contains initial relations of the field scheme used to write the system Lagrangian. It involves the effective potential energy whose quadratic term describes hydrogen bonding between the oligomers and the associative groups of the homopolymer chains, whereas cubic and biquadratic terms relate to the self-action effects. The principal peculiarity of our approach lies in accounting of the inhomogeneity in the distribution of oligomers along the homopolymer chains. This accounting is caused by the introduction of the effective kinetic energy whose density is proportional to the square of the derivative of oligomers distribution over segment numbers $n$. Due to the temperature dependence of the hydrogen coupling, related effective mass is a fluctuating parameter whose averaging, along the Hubbard-Stratonovich procedure, arrives at the biquadratic term with respect to the time derivative. According to the calculations given in Sec. IV, just this term, being considered within the mean-field approach, causes decaying character of the temperature dependence $L(T)$ of the structure period. Complication of the problem arising from the determination of the proper frequency $\omega$ is caused by an essential nonlinearity and coupling the advanced/retarded Green functions and the structure factor. Hence, it is methodically convenient to use the supersymmetry technique that enables to obtain in the simplest way explicit expressions for above functions in the long-range limit (see Sec. III). The divergency condition of the Green function permits to find the proper frequency $\omega$ with accounting self-action effects within supersymmetry perturbation theory. A comparison of the dependencies obtained with experimental data given in Sec. V shows that the scheme developed allows to present in a self-consistent manner the main peculiarities of the microphase separation in the homopolymer-oligomer systems with associative coupling.

\section{GENERIC FORMALISM}

The problem under consideration is addressed to the definition of the effective law of motion $c(n)$ that determines a sequence of oligomer alternation along the homopolymer chain. This is accomplished by means of specifying the occupation number, being $c(n)=1$ if oligomer is attached to the segment $n$ and $c(n)=0$ otherwise (obviously, the mean value $\bar{c} \equiv \overline{c(n)}$ of the occupation number is reduced to $x$ at the oligomer ratio $x \leqslant 1$, while $\bar{c}=1$ for $x>1$ ). When the index of the homopolymer chain $N \rightarrow \infty$, the argument $n$ may be considered as a continuous one, and we are ventured to start with Euler equation [18]

$$
\frac{\delta S}{\delta c}-\frac{d}{d n} \frac{\delta S}{\delta \dot{c}}=\frac{\delta R}{\delta \dot{c}},
$$

where the overdot denotes derivative with respect to the segment number $n$, action $S$ and dissipative functional $R$ take the usual forms

$$
S\{c(n)\} \equiv \int_{0}^{N} L(c(n), \dot{c}(n)) d n, \quad R=\frac{\Theta}{2} \int_{0}^{N}(\dot{c}(n))^{2} d n,
$$

being defined by the Lagrangian $L(c(n), \dot{c}(n))$ and the damping coefficient $\Theta$, respectively. The total action $S=K$ $-\Pi$ is determined by a "kinetic" contribution $K$ of inhomogeneity in the oligomer distribution and "potential" component $\Pi \equiv V_{0}+V$ caused by the interaction between the homopolymer and the oligomer,

$$
V_{0}=T \frac{\tau}{2} \int_{0}^{N}(c(n))^{2} d n
$$

and self-action contribution

$$
V=T \int_{0}^{N} v(c(n)) d n, \quad v \equiv \frac{\mu}{3 !} c^{3}+\frac{\lambda}{4 !} c^{4} .
$$

Here, $T$ is temperature measured in energy units, factor $\tau$ determines the strength of the hydrogen bonding, multipliers $\mu, \lambda$ are self-action parameters.

In comparison with the above standard approach, the determination of the contribution of inhomogeneity along the polymeric chain is a much more delicate problem. Indeed, the bare magnitude can be written in the form of the usual kinetic action

$$
K=T \frac{m}{2} \int_{0}^{N}\left(\frac{d c}{d n}\right)^{2} d n
$$

where an effective mass $m$ appears as a temperature fluctuating parameter with mean value $\bar{m}$ and variance $\overline{(m-\bar{m})^{2}}$ $\equiv \sigma^{2}$ (bar denotes the average, as usual). Then, after averaging exponent $\exp (-K / T)$ over the Gaussian distribution of the bare mass $m$, we obtain the effective kinetic action in the following form:

$$
\begin{gathered}
\mathcal{K}=\bar{K}+\widetilde{K}, \quad \bar{K} \equiv T \frac{\bar{m}}{2} \int_{0}^{N}(\dot{c}(n))^{2} d n, \\
\widetilde{K} \equiv-T \frac{\sigma^{2}}{8} \int_{0}^{N} \int_{0}^{N}(\dot{c}(n))^{2}\left(\dot{c}\left(n^{\prime}\right)\right)^{2} d n d n^{\prime} .
\end{gathered}
$$

As a result, total action takes the final form

$$
\begin{aligned}
S= & T \frac{\bar{m}}{2} \int_{0}^{N}(\dot{c}(n))^{2} d n-T \frac{\sigma^{2}}{8} \int_{0}^{N} \int_{0}^{N}(\dot{c}(n))^{2}\left(\dot{c}\left(n^{\prime}\right)\right)^{2} d n d n^{\prime} \\
& -T \frac{\tau}{2} \int_{0}^{N}(c(n))^{2} d n-V
\end{aligned}
$$

where self-action potential $V$ is given by Eqs. (5). Respectively, Euler equation (2) arrives at the equation of effective motion

$$
(\bar{m}-\widetilde{\Delta}) \ddot{c}+\frac{\Theta}{T} \dot{c}+\tau c=-v^{\prime},
$$


where one notices

$$
v^{\prime} \equiv T^{-1} \frac{\delta V\{c(n)\}}{\delta c(n)}=\frac{\partial v}{\partial c}, \quad \widetilde{\Delta} \equiv \frac{\sigma^{2}}{2} \int_{0}^{N}\left(\dot{c}\left(n^{\prime}\right)\right)^{2} d n^{\prime} .
$$

By introducing the effective mass $\widetilde{m}$, characteristic number of correlating segments $n_{c}$ and $\delta$-correlated source $\zeta(n)$ in accordance with definitions

$$
\begin{gathered}
\widetilde{m} \equiv \bar{m}-\widetilde{\Delta}, \quad n_{c} \equiv \frac{\Theta}{T}, \\
\langle\zeta(n)\rangle=0, \quad\left\langle\zeta(n) \zeta\left(n^{\prime}\right)\right\rangle=\delta\left(n-n^{\prime}\right),
\end{gathered}
$$

one obtains Langevin equation of inertial type,

$$
\widetilde{m} \ddot{c}+n_{c} \dot{c}=-\left(\tau c+v^{\prime}\right)+\zeta .
$$

Making use of the field scheme [11] allows to express the noise $\zeta$ in terms of an additional degree of freedom $p$ being the momentum conjugated to the effective coordinate $c$. Following this line, one has to introduce the generating functional

$$
Z\{c(n)\} \equiv\left\langle\prod_{n} \delta\left\{\tilde{m} \ddot{c}+n_{c} \dot{c}+\tau c+v^{\prime}-\zeta\right\} \operatorname{det}\left|\frac{\delta \zeta}{\delta c}\right|\right\rangle
$$

being the average over the noise $\zeta(n)$ where $\delta$ function accounts for the equation of motion (14), and the determinant is Jacobian of transition from $\zeta(n)$ to $c(n)$ that is equal $\Theta / T \equiv n_{c}$. Then, making use of the functional Laplace representation

$$
\delta\{x(n)\}=\int_{-i \infty}^{i \infty} \exp \left(-\int p x d n\right) D p(n),
$$

over a ghost field $p(n)$ and averaging Eq. (15) over Gaussian distribution

$$
P_{0}\{\zeta(n)\} \propto \exp \left(-\frac{1}{2} \int \zeta^{2}(n) d n\right),
$$

being related to Eqs. (13), we derive to the standard form [11]

$$
\begin{gathered}
Z\{c(n)\}=\int \mathcal{P}\{c(n), p(n)\} \mathrm{D} p(n), \quad \mathcal{P} \equiv e^{-\mathcal{S}} ; \\
\mathcal{S}\{c(n), p(n)\} \equiv \int \mathcal{L}(c(n), p(n)) d n,
\end{gathered}
$$

where effective Lagrangian is introduced,

$$
\mathcal{L}=\left(\widetilde{m} \ddot{c}+n_{c} \dot{c}+\tau c+v^{\prime}\right)\left(n_{c}^{-1} p\right)-\frac{1}{2}\left(n_{c}^{-1} p\right)^{2} .
$$

According to Euler equations [19]

$$
\frac{\partial \mathcal{L}}{\partial x}-\frac{d}{d n} \frac{\partial \mathcal{L}}{\partial \dot{x}}+\frac{d^{2}}{d n^{2}} \frac{\partial \mathcal{L}}{\partial \ddot{x}}=0, \quad x \equiv\{c, p\},
$$

effective motion in the phase space is determined by the system

$$
\begin{gathered}
\tilde{m} \ddot{c}+n_{c} \dot{c}=-\left(\tau c+v^{\prime}\right)+\left(n_{c}^{-1} p\right), \\
\widetilde{m} \ddot{p}-n_{c} \dot{p}=-\left(\tau+v^{\prime \prime}\right) p .
\end{gathered}
$$

A comparison of the first of these equations with Eq. (14) shows that the conjugated momentum $p$ appears as the most probable value of the renormalized noise amplitude $n_{c} \zeta$ [14].

\section{SUPERSYMMETRY REPRESENTATION OF CORRELATION IN OLIGOMER DISTRIBUTION}

Equations (22) and (23) represent a system of nonlinear equations whose solution demands a use of the perturbation theory with respect to the self-action parameters $\lambda, \mu$ and of the self-consistency procedure to determine an effective mass $\widetilde{m}\{c(n)\}$. However, because we are interested in the knowledge not of laws of motion $c(n)$ and $p(n)$, but only of the frequency of oligomer alternation along the homopolymer chain, it is appropriate to restrict ourselves to an investigation of the corresponding correlators. These reduce to autocorrelator, retarded and advanced Green functions defined by the following equalities:

$$
\begin{gathered}
S\left(n-n^{\prime}\right)=\left\langle\delta c(n) \delta c\left(n^{\prime}\right)\right\rangle, \\
G_{-}\left(n-n^{\prime}\right)=\left\langle\delta c(n) p\left(n^{\prime}\right)\right\rangle, \\
G_{+}\left(n-n^{\prime}\right)=\left\langle p(n) \quad \delta c\left(n^{\prime}\right)\right\rangle ; \\
\delta c(n) \equiv c(n)-\bar{c}, \quad \bar{c} \equiv \overline{c(n)},
\end{gathered}
$$

respectively. As is demonstrated in Appendix B, consequent analytical consideration takes a canonical form if one introduces a dual field [12-14]

$$
\phi=\delta c+\left(n_{c}^{-1} p\right) \vartheta,
$$

being built by making use of nilpotent variable $\vartheta$ which satisfies the conditions

$$
\vartheta^{2}=0, \quad \vartheta \vartheta^{\prime}=\vartheta^{\prime} \vartheta, \quad \int d \vartheta=0, \quad \int \vartheta d \vartheta=1 .
$$

As a result, the correlators (24) are reduced to the components of the supercorrelator

$$
C\left(z, z^{\prime}\right) \equiv\left\langle\phi(z) \phi\left(z^{\prime}\right)\right\rangle, \quad z \equiv\{n, \vartheta\}
$$

of the state vectors (25) in the phase space. Indeed, the supercorrelator (27) appears as a pseudovector

$$
\mathbf{C}=G_{+} \mathbf{A}+G_{-} \mathbf{B}+S \mathbf{T}
$$

spanned on set of the orts 
TABLE I. The table of the functional product of vectors (29).

\begin{tabular}{llll}
\hline \hline$l \backslash r$ & $\mathbf{T}$ & $\mathbf{A}$ & $\mathbf{B}$ \\
\hline $\mathbf{T}$ & 0 & $\mathbf{T}$ & 0 \\
$\mathbf{A}$ & 0 & $\mathbf{A}$ & 0 \\
$\mathbf{B}$ & $\mathbf{T}$ & 0 & $\mathbf{B}$ \\
\hline \hline
\end{tabular}

$\mathbf{A}\left(\vartheta, \vartheta^{\prime}\right)=\vartheta, \quad \mathbf{B}\left(\vartheta, \vartheta^{\prime}\right)=\vartheta^{\prime}, \quad \mathbf{T}\left(\vartheta, \vartheta^{\prime}\right)=1$

Introducing the functional product of some vectors $X, Y, Z$ in such a space,

$$
X\left(\vartheta, \vartheta^{\prime}\right)=\int Y\left(\vartheta, \vartheta^{\prime \prime}\right) Z\left(\vartheta^{\prime \prime}, \vartheta^{\prime}\right) d \vartheta^{\prime \prime}
$$

it is easy to see that orts (29) are noncommutative to obey the multiplication rules given in Table I. Thus, making use of the supercorrelator (27) presents a big advantage in analytical calculations.

Under suppression of the inhomogeneity fluctuations along the homopolymer chain $(\sigma=0)$, the action (19) with the Lagrangian (20) written within the harmonic approximation $[v(c)=$ const $]$ takes the canonical form

$$
\mathcal{S}_{0}\{\phi(z)\}=\frac{1}{2} \int \phi(z) L(z) \phi(z) d z
$$

with the linear operator

$$
\begin{gathered}
L(z)=\tau(n)+D(z), \quad \tau(n) \equiv \tau+\bar{m} \partial_{n}^{2}, \quad \partial_{n}^{2}=\frac{\partial^{2}}{\partial n^{2}} \\
D(z)=-\frac{\partial}{\partial \vartheta}+n_{c}\left(1-2 \vartheta \frac{\partial}{\partial \vartheta}\right) \frac{\partial}{\partial n}
\end{gathered}
$$

As shows the consideration in Appendix B, this operator defines the bare supercorrelator

$$
C^{(0)}(z) \equiv L^{-1}(z) \delta\left(\vartheta, \vartheta^{\prime}\right), \quad \delta\left(\vartheta, \vartheta^{\prime}\right)=\vartheta+\vartheta^{\prime}
$$

to be governed by the Dyson equation (54). Taking into account condition (B7), one obtains

$$
C^{(0)}=\frac{[\tau(n)-D] \delta\left(\vartheta, \vartheta^{\prime}\right)}{\tau^{2}(n)-n_{c}^{2} \partial_{n}^{2}} .
$$

Using Fourier transformation over the frequency $\nu$, we obtain the expression

$$
\begin{gathered}
C^{(0)}=\frac{1+\left[\tau(\nu)-i n_{c} \nu\right] \vartheta+\left[\tau(\nu)+i n_{c} \nu\right] \vartheta^{\prime}}{\tau^{2}(\nu)+n_{c}^{2} \nu^{2}}, \\
\tau(\nu) \equiv \tau-\bar{m} \nu^{2}
\end{gathered}
$$

Then, taking into account Eqs. (28) and (29), we get standard equalities for the main correlators

$$
\begin{gathered}
G_{ \pm}^{(0)}=\left[\tau(\nu) \pm i n_{c} \nu\right]^{-1}, \\
S^{(0)} \equiv G_{+}^{(0)} G_{-}^{(0)}=\left[\tau^{2}(\nu)+n_{c}^{2} \nu^{2}\right]^{-1} .
\end{gathered}
$$

An explicit form of linear operator

$$
\mathbf{L}=L_{+} \mathbf{A}+L_{-} \mathbf{B}+L \mathbf{T}
$$

obeying the equality $\mathbf{L} \equiv\left(\mathbf{C}^{(0)}\right)^{-1}$ will be needed below. Using the equality [12]

$$
\mathbf{C}^{-1}=G_{+}^{-1} \mathbf{A}+G_{-}^{-1} \mathbf{B}-G_{+}^{-1} S G_{-}^{-1} \mathbf{T},
$$

we easily obtain the components

$$
L_{ \pm}=\tau(\nu) \pm i n_{c} \nu, \quad L=-1
$$

To proceed, let us consider the effective interaction term in action (9),

$$
\widetilde{K} \equiv-T \frac{\sigma^{2}}{2} \iint \frac{d \nu_{1} d \nu_{2}}{(2 \pi)^{2}} \nu_{1}^{2} \nu_{2}^{2}\left|c\left(\nu_{1}\right)\right|^{2}\left|c\left(\nu_{2}\right)\right|^{2}
$$

taken in the frequency representation. Within the mean-field approximation, one has

$$
\begin{aligned}
\left|c\left(\nu_{1}\right)\right|^{2}\left|c\left(\nu_{2}\right)\right|^{2} & \Rightarrow\left\langle\left|c\left(\nu_{1}\right)\right|^{2}\right\rangle\left|c\left(\nu_{2}\right)\right|^{2}+\left|c\left(\nu_{1}\right)\right|^{2}\left\langle\left|c\left(\nu_{2}\right)\right|^{2}\right\rangle \\
& =S\left(\nu_{1}\right)\left|c\left(\nu_{2}\right)\right|^{2}+\left|c\left(\nu_{1}\right)\right|^{2} S\left(\nu_{2}\right)
\end{aligned}
$$

and the fluctuational component of the inhomogeneity action (41) takes the form

$$
\widetilde{\mathcal{K}}\{\phi\}=-T \Delta \int \frac{d \nu}{2 \pi} \nu^{2}|\phi(\nu, \vartheta)|^{2} \vartheta d \vartheta
$$

where parameter $\tilde{\Delta}$ given by Eq. (11) reduces to averaged magnitude

$$
\Delta=\sigma^{2} \int \frac{d \nu}{2 \pi} \nu^{2} S(\nu) \Rightarrow \sigma^{2} \int \frac{d \nu}{2 \pi} \nu^{2} C(\nu, \vartheta) \vartheta d \vartheta
$$

As a result, the bare mass $\bar{m}$ in the action $\mathcal{S}_{0}$ given by Eqs. (31) and (32) is replaced by the effective quantity

$$
m_{e f} \equiv \bar{m}-\Delta,
$$

being averaged value of the fluctuating mass (12).

To finish supersymmetry representation of the action (19) defined by the Lagrangian (20), one should add to Eqs. (31) and (43) the self-action term

$$
\mathcal{V}\{\phi(z)\}=\int v(\phi(z)) d z, \quad z \equiv\{n, \vartheta\}
$$

with the expansion (5). Then, the standard perturbation theory gives the symbolic expression [11] 
TABLE II. Conventional multiplication table of the Grassman $A, B$ and non-Grassman $T$ quantities [see Eq. (29)].

\begin{tabular}{lccc}
\hline \hline & $T\left(\vartheta, \vartheta^{\prime}\right)$ & $A\left(\vartheta, \vartheta^{\prime}\right)$ & $B\left(\vartheta, \vartheta^{\prime}\right)$ \\
\hline$T\left(\vartheta, \vartheta^{\prime}\right)$ & $T\left(\vartheta, \vartheta^{\prime}\right)$ & $A\left(\vartheta, \vartheta^{\prime}\right)$ & $B\left(\vartheta, \vartheta^{\prime}\right)$ \\
$A\left(\vartheta, \vartheta^{\prime}\right)$ & $A\left(\vartheta, \vartheta^{\prime}\right)$ & 0 & 0 \\
$B\left(\vartheta, \vartheta^{\prime}\right)$ & $B\left(\vartheta, \vartheta^{\prime}\right)$ & 0 & 0 \\
\hline \hline
\end{tabular}

$$
\Sigma\left(\vartheta_{1}, \vartheta_{2}, n\right)=\frac{\mu^{2}}{2 !}\left(C\left(\vartheta_{1}, \vartheta_{2} ; n\right)\right)^{2}+\frac{\lambda^{2}}{3 !}\left(C\left(\vartheta_{1}, \vartheta_{2} ; n\right)\right)^{3}
$$

for the self-energy function $\Sigma\left(\vartheta_{1}, \vartheta_{2}, n\right)$ defined by the following equation for the $n$-point dressed supercorrelator

$$
\begin{aligned}
C^{(n)}\left(\vartheta, \vartheta^{\prime}\right)= & \iint C^{(0)}\left(\vartheta, \vartheta_{1}\right) \Sigma^{(n)}\left(\vartheta_{1}, \vartheta_{2}\right) \\
& \times C^{(0)}\left(\vartheta_{2}, \vartheta^{\prime}\right) d \vartheta_{1} d \vartheta_{2} .
\end{aligned}
$$

However, detailed analysis [17] shows that the multiplication rules given by Table I have to be replaced by the rules of Table II. Then, the components of the pseudovector

$$
\Sigma=\Sigma_{+} \mathbf{A}+\Sigma_{-} \mathbf{B}+\Sigma \mathbf{T}
$$

take the following forms:

$$
\begin{aligned}
\Sigma(\nu)= & \frac{\mu^{2}}{2} \int \frac{d \nu_{1}}{2 \pi} S\left(\nu_{1}\right) S\left(\nu-\nu_{1}\right) \\
& +\frac{\lambda^{2}}{6} \iint \frac{d \nu_{1} d \nu_{2}}{(2 \pi)^{2}} S\left(\nu_{1}\right) S\left(\nu_{2}\right) S\left(\nu-\nu_{1}-\nu_{2}\right) \\
\Sigma_{ \pm}(\nu)= & \mu^{2} \int \frac{d \nu_{1}}{2 \pi} S\left(\nu_{1}\right) G_{ \pm}\left(\nu-\nu_{1}\right) \\
& +\frac{\lambda^{2}}{2} \iint \frac{d \nu_{1} d \nu_{2}}{(2 \pi)^{2}} S\left(\nu_{1}\right) S\left(\nu_{2}\right) G_{ \pm}\left(\nu-\nu_{1}-\nu_{2}\right)
\end{aligned}
$$

Making use of the theory of residues (see Appendix C) with the correlators (37), where the frequency dependent parameter $\tau(\nu) \equiv \tau-\bar{m} \nu^{2}$ is replaced by the bare one $\tau$, one arrives at the equalities (C5) and (C6) which take the form

$$
\begin{aligned}
\Sigma \simeq\left(8 \tau^{3} n_{c}\right)^{-1}\left[\left(\mu^{2}+\frac{\lambda^{2}}{3^{2} \tau n_{c}}\right)-\left(\frac{\mu^{2}}{2^{2}}+\frac{\lambda^{2}}{3^{4} \tau n_{c}}\right) \xi^{2}\right], \\
\Sigma_{ \pm} \simeq\left(4 \tau^{2} n_{c}\right)^{-1}\left[\left(\mu^{2}+\frac{\lambda^{2}}{6 \tau n_{c}}\right) \mp \frac{i}{2}\left(\mu^{2}+\frac{\lambda^{2}}{3^{2} \tau n_{c}}\right) \xi\right. \\
\left.-\left(\frac{\mu^{2}}{2^{2}}+\frac{\lambda^{2}}{2 \times 3^{3} \tau n_{c}}\right) \xi^{2}\right]
\end{aligned}
$$

within the hydrodynamic limit $\xi \equiv \nu / \omega_{s} \ll 1, \omega_{s} \equiv \tau / n_{c}$.

As shows the consideration in Appendix B, self-consistent behavior of the system is described by the generalized Dyson equation

$$
\mathbf{C}^{-1}=\mathbf{L}-\mathbf{\Sigma}
$$

In the component representation this equality arrives at the equations

$$
\begin{gathered}
S=(\Sigma-L) G_{+} G_{-}, \\
G_{ \pm}^{-1}=L_{ \pm}-\Sigma_{ \pm} .
\end{gathered}
$$

Combination of Eqs. (40), (52), and (53) arrives at the final equations for main correlators within the hydrodynamical limit $\xi \ll 1$,

$$
\begin{aligned}
G_{ \pm}^{-1}= & {\left[\tau-\left(4 \tau^{2} n_{c}\right)^{-1}\left(\mu^{2}+\frac{\lambda^{2}}{6 \tau n_{c}}\right)\right] \pm i\left[\tau+\left(8 \tau^{2} n_{c}\right)^{-1}\right.} \\
& \left.\times\left(\mu^{2}+\frac{\lambda^{2}}{3^{2} \tau n_{c}}\right)\right] \xi-\left[\frac{\tau^{2} m_{e f}}{n_{c}^{2}}-\left(4 \tau^{2} n_{c}\right)^{-1}\right. \\
& \left.\times\left(\frac{\mu^{2}}{2^{2}}+\frac{\lambda^{2}}{2 \times 3^{3} \tau n_{c}}\right)\right] \xi^{2}
\end{aligned}
$$

To avoid misunderstanding, we would like to discuss the physical meaning of the limitation caused by the use of the hydrodynamical limit. It might seem that its application creates obstacles in the way of description of the mixtures with high magnitudes of the oligomer concentrations $x \sim 1$. However, it would be right if we have used correlators of the microscopic oligomer numbers $c(n)$ itself, while definitions (24) give the correlators in terms of the deviations $\delta c(n)$ $\equiv c(n)-\bar{c}$ from the mean value $\bar{c}$ [23]. This means we escape the necessity to average over small scales because fluctuations in Eqs. (24) correlate essentially only within weak segregation limit related to the hydrodynamical domain.

\section{DETERMINATION OF THE PERIOD OF MICROPHASE STRUCTURE}

Our consideration is based on the obvious equality for the long space period $L=2 l+D$ where $l$ is the oligomer chain length, $D$ is the thickness of the homopolymer layer being fixed by the inverse share $X^{-1}$ of average number of the hydrogen bonds (see Fig. 1). Physically, this value is reduced to the magnitude $2 \pi / \omega$ determined by the circular frequency $\omega$ in the alternation of the oligomer heads along the homopolymer chain. Then, the long space period is expressed by the following equality [5] (see Appendix A):

$$
L=2 l+D_{0} \omega^{-1}, \quad D_{0} \equiv\left(2 \pi \chi^{1 / 6} n^{-1 / 3}\right) b \geqslant b,
$$

where $\chi \leqslant 10^{-1}$ is the Flory parameter, $n \sim 10$ is the number of segments in oligomer chain, and $b$ is the segment length.

To obtain the frequency $\omega$, one has to determine first the effective mass $m_{e f}$ given by Eqs. (45) and (44). Using the 
theory of residues (see Appendix C) with the structure factor (55) and Green function (57), one arrives at the renormalization mass parameter

$$
\Delta=\frac{\sigma^{2}}{2 m_{e f} n_{c}}\left(1+\frac{1}{2^{5}} \frac{n_{c} \mu^{2}}{\tau^{4} m_{e f}}+\frac{1}{2^{3} \times 3^{4}} \frac{\lambda^{2}}{\tau^{5} m_{e f}}\right),
$$

where only the terms of the second order of smallness over the parameters $\mu, \lambda$ of the self-action (5) are kept. Inserting here Eqs. (12) and (45), we obtain the equations for determination of the effective mass as a function of the temperature:

$$
\begin{gathered}
m_{e f}=\mu \bar{m}, \quad \mu=\mu(T), \\
4 \mu(1-\mu)=\frac{T}{T_{c 0}}\left(1+\frac{\alpha+\beta / T}{\mu}\right),
\end{gathered}
$$

where

$$
T_{c 0} \equiv\left(\frac{\bar{m}}{\sigma}\right)^{2} \frac{\Theta}{2}, \quad \alpha \equiv \frac{1}{2^{3} \times 3^{4}} \frac{\lambda^{2}}{\tau^{5} \bar{m}}, \quad \beta \equiv \frac{1 \Theta \mu^{2}}{2^{5} \tau^{4} \bar{m}} .
$$

Numerical solution of Eq. (61) for different values of $\alpha$ and $\beta$ allows us to estimate the influence of the self-action on the effective mass. It turned out that even small variation of the parameter $\alpha$ substantially changes the shape of the dependence $\mu(T)$, whereas the parameter $\beta$ almost does not affect it, and we can put $\beta=0$ for the sake of simplicity. This means physically that the cubic anharmonicity in the self-action potential energy (5) is irrelevant to the microphase separation phenomenon.

The smallness of the self-action parameters $\alpha, \beta$ allows us to solve Eq. (61) analytically. In doing so, one has to replace the required dependence $\mu(T)$ in the right-hand side of Eq. (61) by the bare dependence

$$
\mu_{0}(T)=\frac{1}{2}\left(1+\sqrt{1-\frac{T}{T_{c 0}}}\right),
$$

which is a solution of this equation at $\alpha=\beta=0$. As a result, we obtain the simple dependence

$$
\mu(T)=\frac{1}{2}\left(1+\sqrt{1-\frac{T}{T_{c}}}\right)
$$

with a characteristic temperature

$$
T_{c} \equiv T_{c 0}(1-2 \alpha),
$$

where the scale $T_{c 0}$ is given by the first of Eqs. (62) [the multiplier should be put $\mu_{0}\left(T_{c 0}\right) \simeq 1 / 2$ due to the smallness of the parameter $\alpha \ll 1]$. According to Eq. (64), with the increase in temperature, the effective mass (60) decreases monotonously from the bare magnitude $\bar{m}$ at $T=0$ to $\bar{m} / 2$ at $T=T_{c}$ (see the main panel in Fig. 2). The critical temperature $T_{c}$ determines the point of the order-disorder transition according to the condition

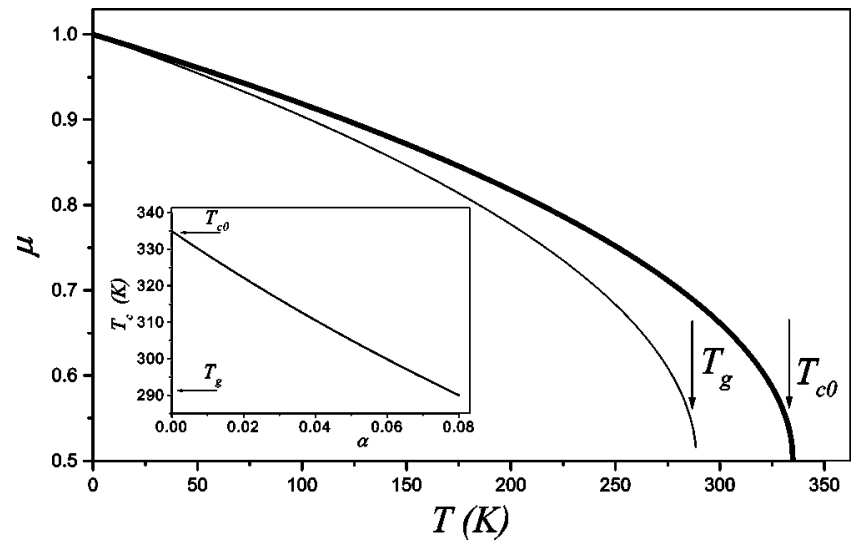

FIG. 2. Temperature dependence of the inhomogeneity parameter $\mu$ for different values of the self-action parameter $\alpha$ : thick curve relates to $\alpha=0$, thin one relates to $\alpha=0.08\left(T_{c 0}=337 \mathrm{~K}\right)$. Inset: the temperature of order-disorder transition $T_{c}$ vs the parameter $\alpha$.

$$
\frac{d \mu}{d T}=-\infty
$$

The resulting dependence $T_{c}$ on the self-action parameter $\alpha$ is shown in the inset of Fig. 2. It is principally important that the bigger the value of the self-action parameter $\alpha$, the more narrow is the temperature domain $T_{g}-T_{c}$ where the microphase separated structure is possible $\left(T_{g}\right.$ being a glassing temperature). In other words, the self-action effect leads to the shrinking of the region of the ordered structure because the critical temperature $T_{c}$ reaches the boundary magnitude $T_{g}$ with increasing of $\alpha$ before the magnitude $\alpha \simeq 0.08$.

The divergency condition $G_{-}^{-1}=0$ of the Green function (57) gives the proper frequency

$$
\nu_{0}= \pm \omega-i \varpi, \quad \omega \equiv \sqrt{\omega_{0}^{2}-\varpi^{2}}
$$

of the oligomer alternation along the homopolymer chain. Real and imaginary parts are determined by the expressions

$$
\begin{aligned}
& \omega_{0} \equiv \frac{\bar{\omega}_{0}}{\sqrt{\mu(T)}}\left[1+3 \alpha\left(1-\frac{9}{8} \frac{T^{2}}{T_{0}^{2}}\right)\right], \\
& \varpi \equiv \frac{\bar{\omega}_{0}}{\mu(T)} \frac{T_{0}}{T}\left[1+6 \alpha\left(1+\frac{3}{8} \frac{T^{2}}{T_{0}^{2}}\right)\right],
\end{aligned}
$$

where the dependence $\mu(T)$ is defined by Eqs. (64) and (65); the effective mass in parentheses after the factor $\alpha \ll 1$ is put to be equal to the value $\bar{m} / 2$ related to the critical temperature $T_{c}$ characteristic scales of both frequency and temperature are introduced as follows:

$$
\bar{\omega}_{0} \equiv \sqrt{\frac{\tau}{\bar{m}}}, \quad T_{0} \equiv \frac{\Theta}{2 \sqrt{\bar{m} \tau}} .
$$

As a result, combination of Eqs. (58), (66), and (67) leads to the final result for the long space period 


$$
\begin{aligned}
L= & 2 l+\frac{\mu(T)}{\sqrt{\mu(T)-\left(T_{0} / T\right)^{2}}} \\
& \times\left[1+\frac{3}{2} \alpha \frac{1+8\left(T_{0} / T\right)^{2}+\frac{9}{4}\left(T_{0} / T\right)^{-2}}{1-2\left(T_{0} / T\right)^{2}}\right] L_{0},
\end{aligned}
$$

where the characteristic length $L_{0} \equiv D_{0} / \bar{\omega}_{0} \sim \sqrt{\bar{m} / \tau} \chi^{1 / 6} b$ $\propto \chi \chi^{1 / 6} N^{1 / 2}$ is the function of both parameters $\chi$ and $N$ being thermodynamically independent. Thus, the first of the exponents in the scaling relation $L_{0} \propto \chi^{a} N^{b}$ takes the magnitude $a=1 / 6$ inherent to the strong segregation regime, whereas the second one $(b=1 / 2)$ is the same for the weak one [20]. Note that the obtained $\chi$ dependence is caused by the multiplier $\chi^{1 / 6}$ in the generic relation (58) that is relevant to the former of the above regimes, while the method developed addresses latter one.

\section{DISCUSSION}

The behavior of the system under consideration is controlled by the parameters $\bar{m}, \tau$, and $\sigma$ which determine the temperature $T_{c}$ of the order-disorder transition and the long space period $L$ given by Eqs. (65) and (69), respectively. Moreover, there is the self-action parameter $0<\alpha \ll 1$ whose value is limited by the magnitude $\alpha_{\max } \simeq 0.08$ (see inset in Fig. 2). To guarantee positive values of the radicand in Eq. (69) at the critical temperature $T_{c}$, the above parameters have to be constrained by the condition

$$
\kappa \geqslant \sqrt{2}
$$

limiting magnitudes of the principal parameter

$$
\kappa \equiv \frac{T_{c}}{T_{0}}=\sqrt{\tau \bar{m}}\left(\frac{\bar{m}}{\sigma}\right)^{2}(1-2 \alpha) .
$$

The minimal magnitude of $\kappa$ fixes the choice of the theory parameters according to the condition

$$
\sigma \leqslant 2^{-1 / 4} \bar{m}^{5 / 4} \tau^{1 / 4}(1-\alpha) .
$$

It would seem from Eqs. (70) and (71) that the decrease of the critical temperature $T_{c}$ with passing from the ionically bonded system [such as P4VP-(DBSA) $)_{x}$ ] to the hydrogen bonded one [e.g., P4VP-(PDP) $)_{x}$ ] is caused only by the growth of the fluctuation parameter $\sigma$ with respect to the mean magnitude of the inhomogeneity parameter $\bar{m}$. It appears, however, that the main reason for such behavior is given by the decrease of the mean-geometrical magnitude $\sqrt{\bar{m} \tau}$ of the principal coefficients in the generic Lagrangian (9) (see below).

To clarify this problem and find explicit form of the dependencies of the temperature of order-disorder transition $T_{c}$ and the period $L$ on the oligomeric fraction $x$, we assume for main theory parameters $\bar{m}$ and $\tau$ the three-parametric relations,

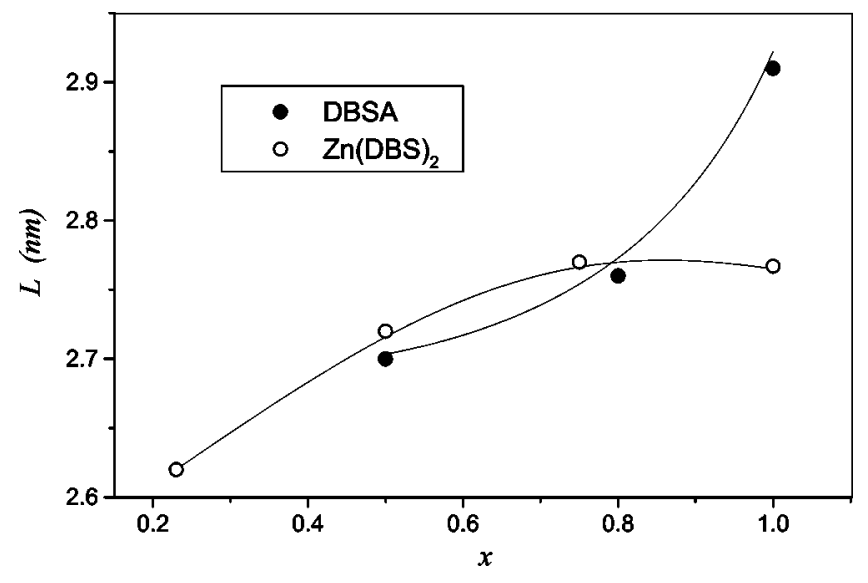

FIG. 3. Long space period in the strongly bonded systems as a function of the oligomeric fraction $x$. Solid lines represent the results of fitting in accordance with Eq. (69). Experimental data for P4VP-(DBSA $)_{x}(\mathbf{O})$ and P4VP-[Zn(DBS $\left.)_{2}\right]_{x}(\bigcirc)$ at room temperature are taken from Ref. [5].

$$
\bar{m}=m_{0}+A x\left(x_{m}-x\right), \quad \tau=\tau_{0}+B x\left(x_{\tau}-x\right)
$$

with positive constants $m_{0}, \tau_{0}, A, B, x_{m}, x_{\tau}$ to be determined. Then, the fitting of the experimental results shown in Fig. 3 in accordance with Eq. (69) where $\tau, \bar{m}$ are given by Eq. (73) leads to the following results for the ionically bonded systems.

The mixtures P4VP-(DBSA) $)_{x}$ :

$$
\begin{gathered}
m_{0}=18, \quad A=8, \quad x_{m}=1.5 ; \\
\tau_{0}=0.6, \quad B=1.5, \quad x_{\tau}=1.0 ; \\
\alpha=0.01, \quad b=1 \mathrm{~nm}, \quad l=10 \mathrm{~nm} .
\end{gathered}
$$

The mixtures P4VP-[Zn(DBS $\left.)_{2}\right]_{x}$ :

$$
\begin{gathered}
m_{0}=5.3, \quad A=26, \quad x_{m}=1.6 ; \\
\tau_{0}=0.8, \quad B=0.1, \quad x_{\tau}=1.0 ; \\
\alpha=0.01, \quad b=1 \quad \mathrm{~nm}, \quad l=10 \mathrm{~nm} .
\end{gathered}
$$

At $x=1$ one obtains $\bar{m}=22, \tau=0.6$ for P4VP-(DBSA) $)_{x}$ and $\bar{m}=20.9, \tau=0.8$ for P4VP- $\left[\mathrm{Zn}(\mathrm{DBS})_{2}\right]_{x}$. Then, Eq. (71) gives values $\kappa=10^{3}, 10^{2}$ at $\sigma=1.31,4.18$, respectively.

A much more complicated situation occurs in the weakly bonded system P4VP-(PDP) $)_{x}$. Here, decrease of the parameter (71) results in the narrowing of the temperature domain $T_{0}-T_{c}$ of the phase separation. All parameters for this class of systems can be determined by the combined fitting of a series of experimental data for the critical temperature $T_{c}$ and the long space period $L$ (see Figs. 4-6). First constraints follow from the comparison of experimental points for the temperature $T_{c}$ of order-disorder transition (see Fig. 4) with fitting results based on Eq. (65) at $\alpha=0.01, l=10 \mathrm{~nm}$ : 


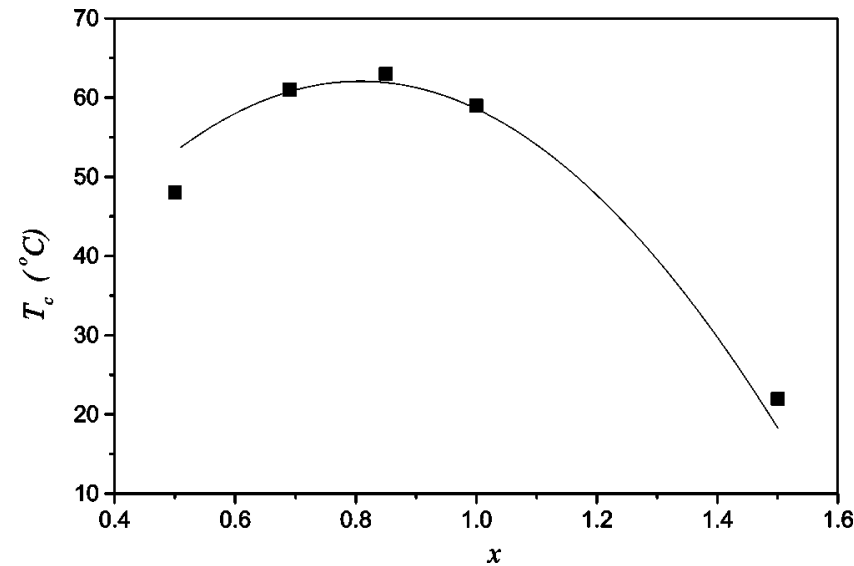

FIG. 4. Order-disorder transition temperature $T_{c}$ for the weakly bonded system as a function of the oligomeric fraction $x$. The solid line represents the dependence obtained by fitting according to Eq. (65). Experimental data for P4VP-(PDP $)_{x}(\boldsymbol{\square})$ are taken from Ref. [4].

$$
\frac{\Theta m_{0}^{2}}{\sigma^{2}}=562, \frac{A}{m_{0}}=0.155, x_{m}=1.615 .
$$

The following parameters gives application of Eq. (69) for the long space period at the temperature $T=T_{c}$ to the data shown in Fig. 5 as the nonmonotonous curve:

$$
\frac{m_{0}}{\tau_{0}}=1499, \quad \frac{B}{\tau_{0}}=7.968, \quad x_{\tau}=1.926 .
$$

Finally, making use of the expression (69) and experimental data for the temperature dependence of the long space period given in Fig. 6 yields the last constraint

$$
\frac{\Theta}{\sqrt{m_{0} \tau_{0}}}=924
$$

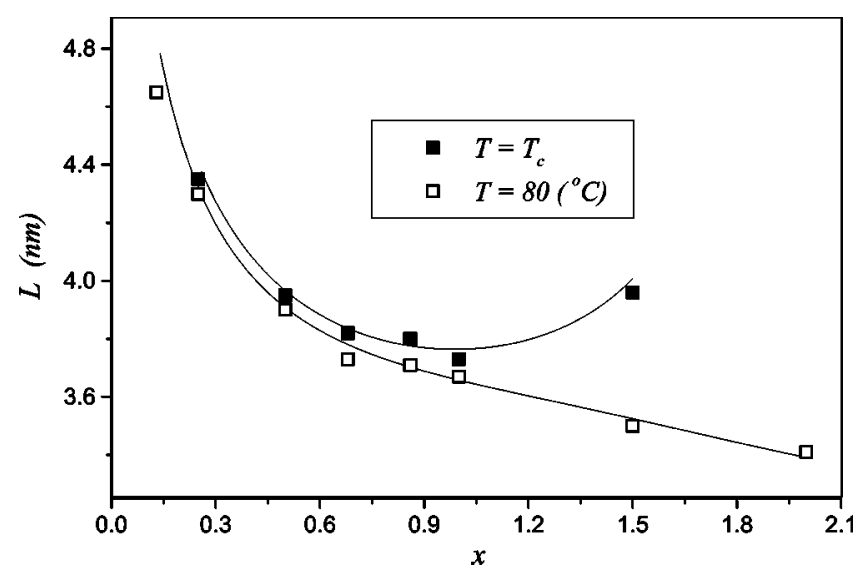

FIG. 5. Long space period in the weakly bonded systems as a function of the oligomeric fraction $x$. Solid lines represent the results of fitting in accordance with Eq. (69). Experimental data for P4VP-(PDP $)_{x}$ at temperature of order-disorder transition $T_{c}$ and at temperature $T=80^{\circ} \mathrm{C}(\square)$ are taken from Ref. [4].

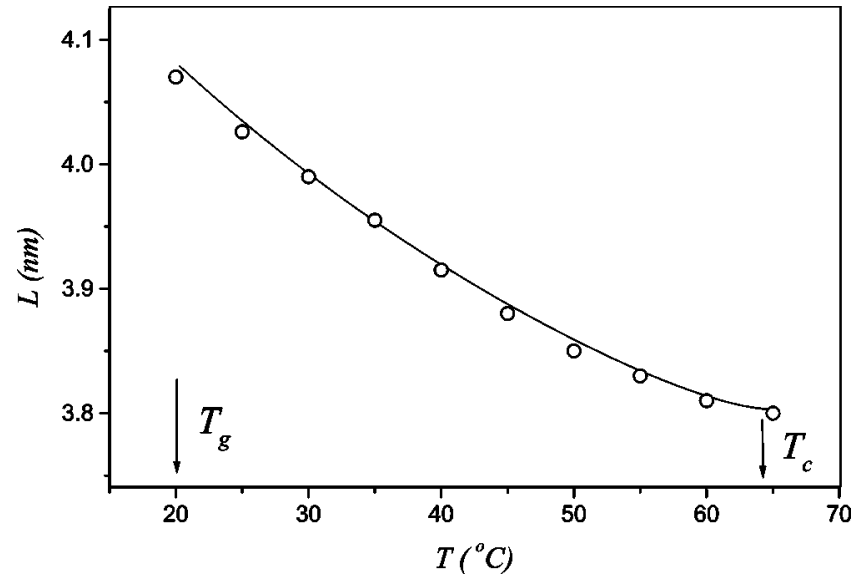

FIG. 6. Temperature dependence of the long space period in the weakly bonded system. The solid line represents the dependence obtained by fitting according to Eq. (69). Experimental data for P4VP-(PDP) $)_{x}$ at $x=0.85(\mathrm{O})$ are taken from Ref. [4].

As a result, taking $m_{0}=1$ at $x=1$ the magnitudes $A$ $=0.155, \Theta=23.87, \sigma=0.206$ are obtained to provide an extremely small value $\tau=5.6 \times 10^{-3}$ of the hydrogen bonding strength and the temperature scale $T_{0}=160$. At $\alpha$ $=0.01$ this arrives at the rest of the parameters $\kappa=2.07, \lambda$ $=6.22 \times 10^{-6}$.

It is worthwhile to discuss separately the dependence of the long space period on the oligomer/monomer ratio at the temperature $T=80^{\circ} \mathrm{C}$ that relates to the monotonous decaying curve shown in Fig. 5. Because the maximal temperature of the order-disorder transition is $T_{c} \approx 65^{\circ} \mathrm{C}$, corresponing to $x=0.85$ (see Fig. 4), experimental data related to $T=80^{\circ} \mathrm{C}$ are obtained for the temperature being beyond of the region of the ordered structure $\left(T>T_{c}\right)$. From the physical point of view, at the critical temperature $T=T_{c}$ the periodicity of the microphase separated structure formed is caused by longrange correlations, whereas at $T=80^{\circ} \mathrm{C}$ only short-range correlations hold to be determined by the homopolymer backbone together with the hydrogen bound surfactant molecules [4]. Fitting of the experimental points for the dependence $L(x)$ at the temperature $T=80^{\circ} \mathrm{C}$ can be done on the basis of Eq. (69) where one puts $\mu(T)=\mu\left(T_{c}\right)=1 / 2$. Then, the values of the parameters obtained differ from those obtained for $T=T_{c}$ by the following constraints:

$$
\frac{B}{\tau_{0}}=4.2, \quad x_{\tau}=3.27, \frac{\Theta}{\sqrt{m_{0} \tau_{0}}}=543 .
$$

Obviously, this difference is due to the temperature dependence of the hydrogen bonding parameter $\tau$ in the potential energy (4).

To conclude our estimations, we notice that the model developed explains successfully a vast variety of peculiarities obtained experimentally for various classes of homopolymer-oligomer mixtures with the interactions of different strength. The data obtained for strong, intermediate, and weak coupled systems P4VP-(DBSA) $)_{x}$ P4VP$\left[\mathrm{Zn}(\mathrm{DBS})_{2}\right]_{x}$ and $\mathrm{P} 4 \mathrm{VP}-(\mathrm{PDP})_{x}$, respectively, are given in 
TABLE III. Theoretical results for three types of homopolymeroligomer systems involving weak, intermediate, and strong interactions (see the text).

\begin{tabular}{lccc}
\hline \hline & P4VP-(DBSA $)_{x}$ & P4VP-[Zn(DBS $\left.)_{2}\right]_{x}$ & P4VP-(PDP $)_{x}$ \\
\hline$m_{0}$ & 18 & 5.3 & 1.000 \\
$A$ & 8 & 26 & 0.155 \\
$x_{m}$ & 1.5 & 1.6 & 1.615 \\
$\bar{m}$ & 22 & 20.9 & 1.095 \\
$\tau_{0}$ & 0.6 & 0.8 & $6.67 \times 10^{-4}$ \\
$B$ & 1.5 & 0.1 & $5.3 \times 10^{-3}$ \\
$x_{\tau}$ & 1.0 & 1.0 & 1.926 \\
$\tau$ & 0.6 & 0.8 & $5.6 \times 10^{-3}$ \\
$\sigma$ & 1.31 & 4.18 & 0.206 \\
$\alpha$ & 0.01 & 0.01 & 0.01 \\
$\lambda$ & 3.33 & 6.66 & $6.22 \times 10^{-6}$ \\
$\Theta(\mathrm{K})$ & $10^{3}$ & $10^{3}$ & 23.87 \\
$T_{c 0}(\mathrm{~K})$ & $1.41 \times 10^{5}$ & $1.25 \times 10^{4}$ & 337 \\
$T_{c}(\mathrm{~K})$ & $1.38 \times 10^{5}$ & $1.22 \times 10^{4}$ & 331 \\
$T_{0}(\mathrm{~K})$ & 138 & 122 & 160 \\
$\kappa$ & $10^{3}$ & $10^{2}$ & 2.07 \\
$l(\mathrm{~nm})$ & 10 & 10 & 10 \\
$b(\mathrm{~nm})$ & 1 & 1 & 1 \\
\hline \hline
\end{tabular}

Table III. It is seen that the coupling weakening gives rise to a decrease of both inhomogeneity parameters $\bar{m}$ and $\sigma$, as well as the crucial decrease of the hydrogen bonding parameter $\tau$ and the self-action parameter $\lambda$, on the one hand, and the characteristic temperatures $T_{c}$ and $\Theta$, on the other hand. According to the relations (71) this leads to extremely large suppression of the value of the parameter $\kappa$ that causes the crucial shrinking the temperature interval of the microphase separation. An analogous effect is caused by the self-action increase.

To avoid misunderstanding, we would like to stress a composite character of the approach used. As it is mentioned in Introduction, this circumstance is expressed by dividing the total free energy (1) into two terms, the first one $F_{h o}$ is relevant to the nonassociated homopolymer-oligomer mixture, the second one $F_{h b}$ is caused by the hydrogen bonding. These terms are caused by the interactions of principally different physical nature: the behavior of the mixture of nonassociated homopolymers and oligomers is determined by the Flory parameter $\chi$, characterizing unfavorable interactions between the oligomers and the rest of the system; the temperature induced distribution of hydrogen bonds is determined by the parameter $\tau$, giving the strength of this bonding. From the formal point of view, both of the above contributions $F_{h o}(\chi, \phi)$ and $F_{h b}(\tau, x)$ should have similar dependencies on the state parameters being (apart from the temperature) the volume fraction of the homopolymer $\phi$ for the first contribution, and the oligomer/monomer ratio $x$ for the second one. Because the term $F_{h o} \sim \chi \phi(1-\phi)$ involves the parabolic dependence on the parameter $\phi$ bounded by maximal value $\phi=1$, we took generalized parabolic approximation (73) for the dependence of the hydrogen bonding strength $\tau$ on the oligomer/monomer ratio $x$ which may take values $x>1$.
Apart from the above difference in the nature of the interactions, one needs to emphasize at once the difference in the approaches used: the mixture of nonassociated homopolymers and oligomers had been studied within the strong segregation limit [5], whereas for the consideration of the hydrogen bonding we use the opposite approach. This difference is kept if the Flory parameter takes large values $\chi \leqslant 10^{-1}$, whereas the hydrogen bonding strength is relatively small $(\tau \ll 1)$. Indeed, the formula (58) for the long space period was obtained within the approximation of the sharp interface, whose thickness is $\Delta \sim \chi^{-1 / 2} b \geqslant 3 b$ to be relevant to the strong segregation regime [5]. In the consideration presented, we have focused mainly on the study of the hydrogen bonding on the base of the action (9) that has the form of series in powers of the order parameter $c$ and its derivatives $\dot{c}$. Such an expansion supposes making use of the weak segregation limit corresponding to the small values of the parameters $\bar{m}$ and $\tau$.

Finally, it is worthwhile to discuss a difference with an usual picture of the phase transitions that is caused by the self-consistency condition (44). A critical value of the Flory parameter $\chi_{c}$ in usual copolymers is known to be caused by the self-action effects. Accounting for these effects involves replacement of the bare parameter $\chi$ by the renormalized value $\chi-\chi_{c}$ [10]. However, in our case the value of Flory parameter is so large that the temperature of the separation of nonassociated polymer-oligomer mixture is negligibly small. As a result, the role of $\chi$ passes to the hydrogen bonding parameter $\tau$ which does not relate to the tendency of monomers of the different kinds to avoid each other. However, as is shown by the considerations given in Refs. [6,7], understanding of the whole picture of microphase separation, including the temperature dependence of the structure period, demands accounting for the inhomogeneity in the distribution of oligomers along homopolymer chains. Within the approach developed, this is reached by means of the effective kinetic energy (6), with the mass fluctuating due to the temperature dependence of hydrogen bonding. This dependence leads to the reduction (45) of the effective mass $m_{e f}$ that causes a phase transition from stochastic to periodic distribution of the oligomers along the homopolymer chain. However, if the critical point is fixed usually by the condition $m_{e f}=0$ [11], in our case the critical temperature $T_{c}$ relates to the finite magnitude $m_{e f}=\bar{m} / 2$ of the effective mass which has a singularity $d m_{e f} / d T=-\infty$ in the temperature derivative (see Fig 2).

\section{ACKNOWLEDGMENTS}

Financial support by the Grant Agency of the Czech Republic (Grant No. GAČR 203/02/0653) is gratefully acknowledged.

\section{APPENDIX A: DERIVATION OF A GENERIC RELATION FOR MICROPHASE STRUCTURE PERIOD}

Following Ref. [5] we suppose the period of the microphase structure to be determined by the minimum of the specific free energy 


$$
f \equiv \frac{1}{V} \frac{F_{i n t}+F_{s t r}}{T}, \quad V \equiv L S, \quad L \equiv 2 l+D
$$

related to the first term in Eq. (1). Being the free energy of the homopolymer-oligomer mixture, this term consists of the interfacial and stretching components $F_{\text {int }}, F_{\text {str }}$ measured in the temperature units $T$ per domain volume $V$ (according to Fig. $1 L, l$, and $D$ are the long space period, the length of the oligomer tail, and the thickness of the homopolymer layer, respectively; $S$ is the domain surface area).

The interfacial free energy is stipulated by the loss of conformational entropy caused by the localization of the homopolymer chains within the interface of thickness $\Delta$. Due to unfavorable interaction $\chi$ between the oligomer tails and the polymer layer the chains form up loops containing segments of number $\mathcal{N}_{s} \sim \chi^{-1}$ [21]. Then, within the model of the random walk, the interface thickness is estimated by the relations $\Delta^{2} \simeq \mathcal{N}_{s} b^{2} \sim b^{2} / \chi$ where $b$ is the segment length. Respectively, the interfacial free energy $F_{\text {int }} \simeq \mathcal{N}_{l} T$ is determined by the number $\mathcal{N}_{l} \simeq S \Delta / \mathcal{N}_{s} b^{3}$ of the loops within the interface. As a result, we obtain the estimation

$$
F_{i n t} \sim \frac{\chi^{1 / 2}}{b^{2}} T S
$$

Another addition $F_{\text {str }}$ is caused by the stretching of the surfactant side chains, whereas the stretching of the homopolymer chains enlarges only the volume part of the free energy. This addition is expressed by the simple equality $F_{s t r} \simeq \mathcal{N}_{c} n_{s} F_{1}$ where the first factor $\mathcal{N}_{c} \simeq D S / N b^{3}$ gives the number of chains per layer, the second multiplier $n_{s}$ $\simeq(b / \lambda) N$ is the number of the oligomer molecules per chain of $N$ segments ( $\lambda$ is period of the oligomers alternating along the chain) and the last factor $F_{1} \sim\left(l^{2} / n b^{2}\right) T$ presents the free energy of stretching a side chain of $n$ segments to a length $l$. Combining the above multipliers, we find the estimation for the total free energy of stretching

$$
F_{s t r} \sim \frac{l^{2} D S}{n b^{4} \lambda} T
$$

To derive the explicit expression for the dependence of the free energy (A1) on the layer thickness $D$ we need to use an obvious condition $2 l S \equiv \mathcal{N}_{c} n_{s} v_{s}$ where $v_{s}=n b^{3}$ is the volume of the surfactant molecule. As a result, we obtain the relation

$$
\frac{2 l}{D} \equiv \frac{b}{\lambda} n
$$

according to which the period $\lambda$ defines the rest of the geometrical characteristics of the microphase separated structure. Inserting Eqs. (A2)-(A4) into Eq. (A1), we obtain the final expression for the interfacial free energy:

$$
f \sim \frac{\frac{\chi^{1 / 2}}{D}+\frac{n}{\lambda^{3}} D^{2}}{[1+(b / \lambda) n] b^{2}},
$$

where numerical coefficients are dropped. The minimization condition $\partial f / \partial D=0$ arrives at the steady-state values of the homopolymer layer thickness and the oligomer length

$$
D \sim \frac{\chi^{1 / 6}}{n^{1 / 3}} \lambda, \quad 2 l \sim\left(\chi^{1 / 6} n^{2 / 3}\right) b .
$$

It is convenient to express the above results by means of the dimensionless frequency of the oligomer alternating along the homopolymer chain:

$$
\omega \equiv \frac{2 \pi}{\lambda / b}=\omega_{\max } X, \quad \omega_{\max } \equiv 2 \pi, \quad X \equiv \frac{b}{\lambda},
$$

where $X$ is the averaged oligomeric fraction per homopolymer. So, the long space period of the microphase separated structure takes the form

$$
L \equiv 2 l+D \simeq \chi^{1 / 6} n^{-1 / 3}\left(n+\frac{2 \pi}{\omega}\right) b .
$$

\section{APPENDIX B: INTRODUCTION TO THE SUPERSYMMETRY FORMALISM}

By analogy with the complex calculus, known convenience of the supersymmetrical calculus is based on a possibility to lick relevant expressions into a canonical shape [11]. In so doing, we write out in the Lagrangian (20) of the Euclidean field theory the kinetic $\kappa$ and the potential $\pi$ energies:

$$
\begin{gathered}
\mathcal{L}=\kappa+\pi, \\
\kappa \equiv\left(\tilde{m} \ddot{c}+n_{c} \dot{c}+\tau c\right)\left(n_{c}^{-1} p\right)-\frac{1}{2}\left(n_{c}^{-1} p\right)^{2}, \\
\pi \equiv n_{c}^{-1} \frac{\partial v}{\partial c} p .
\end{gathered}
$$

To derive the kinetic energy in the canonical form type of Eq. (31),

$$
\kappa=\frac{1}{2} \int \phi(\vartheta) L(\vartheta) \phi(\vartheta) d \vartheta, \quad L \equiv\left(\tau+\tilde{m} \frac{\partial^{2}}{\partial n^{2}}\right)+D,
$$

we need to find the supersymmetry generator $D=D(\vartheta)$. In general case, it is represented in the following way:

$$
D \equiv a+b \frac{\partial}{\partial \vartheta}+c \vartheta+d \vartheta \frac{\partial}{\partial \vartheta}
$$

where the coefficients $a, b, c, d$ are functions of the derivative $\partial / \partial n$. Substitution of expressions (25) and (B5) into Eq. 
(B4), taking into account Eqs. (26), leads to the required expression (B2) with the values

$$
a=n_{c} \frac{\partial}{\partial n}, \quad b=-1, \quad c=0, d=-2 n_{c} \frac{\partial}{\partial n} .
$$

As a result, operator (B5) takes the form (33). It is easy to prove that this operator is Hermitian and has the property

$$
D^{2}=n_{c}^{2} \frac{\partial^{2}}{\partial n^{2}} .
$$

To obtain the nilpotent form

$$
\pi=\frac{1}{2} \int v(\phi(\vartheta)) d \vartheta
$$

of the potential energy (B3), we expand the potential $v(\phi)$ at hand in powers of the term $\left(n_{c}^{-1} p\right) \vartheta$,

$$
\pi=\frac{1}{2} \int\left[v(c)+\left(n_{c}^{-1} \frac{\partial v}{\partial c} p\right) \vartheta\right] d \vartheta
$$

Here, all terms of higher order vanish because of the nilpotency condition. Given the properties of equalities (26), the integration in Eq. (B9) leads to the desired result (B3).

When an infinitesimal increment $\delta \phi$ is added to the field $\phi$, the action related to the Lagrangian (B1) with components (B4) and (B8) acquires the zeroth addition if supersymmetric Euler equation is valid,

$$
L \frac{\delta \mathcal{L}}{\delta(L \phi)}+\frac{\delta \mathcal{L}}{\delta \phi}=0 .
$$

Substituting here the expressions (B4) and (B8), we arrive at the supersymmetric equation of motion

$$
L \phi(z)+\frac{\delta \pi}{\delta \phi(z)}=0
$$

that is reduced to the system (22), (23). Multiplying Eq. (B11) by the field $\phi\left(z^{\prime}\right)$ and averaging the result, we arrive at the Dyson equation (54) where the self-energy function is defined by the equality

$$
\Sigma\left(z, z^{\prime}\right)=-\int\left\langle\frac{\delta \pi}{\delta \phi(z)} \phi\left(z^{\prime \prime}\right)\right\rangle C^{-1}\left(z^{\prime \prime}, z^{\prime}\right) d z^{\prime \prime} .
$$

For performing immediate calculations it is much more convenient to use the standard perturbation theory [11] that arrives at the expressions (50), (51).

It is worthwhile to note finally that the above Jacobian reduced in Eq. (15) to the constant value $n_{c}$ is relevant to the weak segregation limit. Indeed, in the opposite case a passage to the canonical representation type of Eq. (18) demands introducing Grassmannian fields $\bar{\psi}(n), \psi(n)$ that expand the dual field (25) to the four-component form [11]

$$
\Phi=\delta c+\bar{\theta} \psi+\bar{\psi} \theta+\left(n_{c}^{-1} p\right) \bar{\theta} \theta,
$$
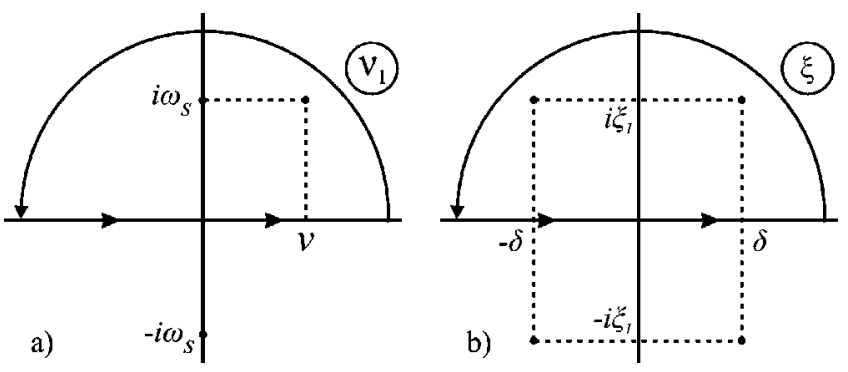

FIG. 7. Poles of the convolution integral in Eqs. (C2)(a) and (C7)(b). $\delta \equiv \sqrt{\xi_{2}^{2}-\xi_{1}^{2}}$.

where anticommutating Grassmannian variables $\bar{\theta}, \theta$ are connected with the commutating nilpotent one $\vartheta$ by means of the equality $\vartheta \equiv \bar{\theta} \theta$. In such a case, the equations of motion (22) and (23) are supplemented with two equations for the conjugate fields $\bar{\psi}(n), \psi(n)$ whose combination arrives at the continuity equation for a quantity $\mathcal{N} \equiv\langle\bar{\psi} \psi\rangle$ playing the role of the density of interface boundaries [14]. Thus, we can conclude that the weak segregation limit related to the density $\mathcal{N}=0$ means suppression of the Grassmannian components reducing the four-component supersymmetrical field (B13) to the two-component dual form (25).

\section{APPENDIX C: CALCULATIONS OF CONVOLUTION INTEGRALS}

a. Self-energy functions. Calculations of the self-energy functions (50), (51) lead to a rather tedious procedure due to the convolution integrals. To demonstrate the line of these calculations we consider in detail the simplest integral related to the first term in Eq. (51),

$$
\Sigma_{-}^{\mu}(\nu)=\mu^{2} \int \frac{d \nu_{1}}{2 \pi} S\left(\nu_{1}\right) G_{-}\left(\nu-\nu_{1}\right) .
$$

Making use of the expressions (37) for the structure factor $S$ and Green function $G_{-}$, where the frequency dispersed parameter $\tau(\nu)$ is replaced by its bare magnitude $\tau$, one arrives at the convolution integral

$$
\Sigma_{-}^{\mu}(\nu)=\frac{\mu^{2}}{2 \pi n_{c}^{3}} \int \frac{d \nu_{1}}{\left(\omega_{s}^{2}+\nu_{1}^{2}\right)\left[\omega_{s}-i\left(\nu-\nu_{1}\right)\right]},
$$

where a characteristic frequency $\omega_{s} \equiv \tau / n_{c}$ is introduced. This integral has the poles $\pm i \omega_{s}$ and $\nu+i \omega_{s}$ [see Fig. 7(a)]. In accordance with the theory of residues [22], the integral in Eq. (C2) is reduced to sum over two of these residues that locate in upper half-plane of the complex frequency $\nu_{1}$,

$$
2 \pi i\left[\frac{-i}{2 i \omega_{s}(-\nu)}+\frac{-i}{\nu\left(\nu+2 i \omega_{s}\right)}\right],
$$

where terms in the square brackets relate to the poles $i \omega_{s}$ and $\nu+i \omega_{s}$, respectively. After a simple algebra this expression yields 


$$
\Sigma_{-}^{\mu}=\frac{\mu^{2}}{\tau^{2} n_{c}} \frac{1+\frac{i}{2} \xi}{4+\xi^{2}}, \quad \xi \equiv \frac{\nu}{\omega_{s}} .
$$

Analogously, the rest of convolution integrals are calculated giving final form of Eqs. (50) and (51):

$$
\begin{gathered}
\Sigma=\frac{\mu^{2}}{2 \tau^{3} n_{c}} \frac{1}{4+\xi^{2}}+\frac{\lambda^{2}}{8 \tau^{4} n_{c}^{2}} \frac{1}{9+\xi^{2}}, \\
\Sigma_{ \pm}=\frac{\mu^{2}}{\tau^{2} n_{c}} \frac{1 \mp \frac{i}{2} \xi}{4+\xi^{2}}+\frac{\lambda^{2}}{8 \tau^{3} n_{c}^{2}} \frac{3 \mp i \xi}{9+\xi^{2}} .
\end{gathered}
$$

b. Renormalization mass parameter. Explicit form of the renormalization mass parameter (44) is determined by the structure factor (55) and Green function (57) with the effective mass (45) and parameter $\tau(\nu)$ being replaced by bare $\tau$,

$$
\Delta=\frac{\sigma^{2}}{\pi n_{c} m_{e f}} \int \frac{\left(\xi_{1}-\xi_{0}^{-1} \xi^{2}\right) \xi^{2} d \xi}{\left(\xi^{2}-2 i \xi_{1} \xi-\xi_{2}^{2}\right)\left(\xi^{2}+2 i \xi_{1} \xi-\xi_{2}^{2}\right)},
$$

where one denotes

$$
\begin{gathered}
\xi_{0} \equiv \frac{16 m_{e f} \tau}{n_{c}}\left(\frac{\mu^{2}}{4}+\frac{\lambda^{2}}{3^{4} \tau n_{c}}\right)^{-1}, \\
\xi_{1} \equiv \frac{n_{c}^{2}}{2 m_{e f} \tau}\left[1+\frac{1}{8 \tau^{3} n_{c}}\left(\mu^{2}+\frac{\lambda^{2}}{9 \tau n_{c}}\right)\right], \\
\xi_{2}^{2} \equiv \frac{n_{c}^{2}}{m_{e f} \tau}\left[1-\frac{1}{4 \tau^{3} n_{c}}\left(\mu^{2}+\frac{\lambda^{2}}{6 \tau n_{c}}\right)\right] .
\end{gathered}
$$

The integral in Eq. (C7) has the pole structure that is shown in Fig. 7(b). As above, the sum over residues located in the upper half-plane of the complex frequency $\xi$ yields the integral value

$$
\frac{\pi}{2}\left(1-\frac{\xi_{2}^{2}-4 \xi_{1}^{2}}{\xi_{0} \xi_{1}}\right)
$$

Accounting for the notices (C8) and keeping only the terms of the second order of smallness over parameters $\mu$ and $\lambda$, one obtains the final expression (59).
[1] M. Antonietti, A. Wenzel, and A. Thunemann, Langmuir 12, 2111 (1996).

[2] O. Ikkala, J. Ruokolainen, G. Ten Brinke, M. Torkkeli, and R. Serimaa, Macromolecules 28, 7088 (1995).

[3] J. Ruokolainen, G. Ten Brinke, O.T. Ikkala, M. Torkkeli, and R. Serimaa, Macromolecules 29, 3409 (1996).

[4] J. Ruokolainen et al., Phys. Rev. E 54, 6646 (1996).

[5] G. Ten Brinke, J. Ruokolainen, and O. Ikkala, Europhys. Lett. 35, 91 (1996).

[6] F. Tanaka and M. Ishida, Macromolecules 30, 1836 (1997).

[7] E. Dormidontova and G. Ten Brinke, Macromolecules 31, 2649 (1998).

[8] L. Leibler, Macromolecules 13, 1602 (1980).

[9] G.N. Fredrickson, S.T. Milner, and L. Leibler, Macromolecules 25, 6341 (1992).

[10] C.D. Sfatos and E.I. Shakhnovich, Phys. Rep. 288, 77 (1997).

[11] J. Zinn-Justin, Quantum Field Theory and Critical Phenomena (Clarendon Press, Oxford, 1993).

[12] A.I. Olemskoi, Physica A 270, 444 (1999).

[13] A.I. Olemskoi and V.A. Brazhnyi, Physica A 273, 368 (1999).

[14] A.I. Olemskoi, Usp. Fiz. Nauk. 171, 503 (2001) [Phys. Usp.
44, 479 (2001)].

[15] T.A. Vilgis, J. Phys. A 24, 5321 (1991).

[16] P.C. Martin, E.D. Siggia, and H.A. Rose, Phys. Rev. A 8, 423 (1973).

[17] J. Kurchan, J. Phys. I 2, 1333 (1992).

[18] L. D. Landau and E. M. Lifshitz, Statistical Physics, Part 1 (Pergamon Press, Oxford, 1980).

[19] A. I. Olemskoi, Theory of Structure Transformations in NonEquilibrium Condensed Matter (NOVA Science, New York, 1999).

[20] K.R. Shull, Macromolecules 25, 2122 (1992).

[21] E. Helfand and Y. Tagani, J. Polym. Sci., Part B: Polym. Lett. 9, 799 (1971).

[22] R. Remmert, Theory of Complex Functions (Springer, New York, 1991).

[23] Strictly speaking, the above definition $\delta c(n) \equiv c(n)-\bar{c}$ of fluctuations is applicable to the disordered phase only ( $T$ $\left.>T_{c}\right)$. Below the critical temperature $\left(T<T_{c}\right)$, one needs to pass to the frequency representation $\delta c(\nu) \equiv c(\nu)-\bar{c} \delta(\nu$ $\left.-\nu_{0}\right)$ where the proper frequency $\nu_{0} \equiv 2 \pi(b / \lambda)$ is relevant to the period $\lambda$ of the oligomer alternation in the ordered phase. 